\title{
Thickness Anomalies in Supported Polystyrene Films with Thicknesses Comparable to the Radius of Gyration
}

\author{
By Chunming YANG, Amane KITAHARA, and Isao TAKAHASHI*
}

\begin{abstract}
We report on X-ray reflectivity of ultrathin polystyrene films with thickness $h$, which is comparable to the radius of gyration $R_{\mathrm{g}}$. Tiny jumps in thickness, accompanying an increase in surface roughness and decrease in electron density, are reproductively observed at around $80{ }^{\circ} \mathrm{C}$ and $92{ }^{\circ} \mathrm{C}$ only with increasing temperature. For films thinner than $R_{\mathrm{g}}$, the jump at $92{ }^{\circ} \mathrm{C}$ disappears. However, films of $2 R_{\mathrm{g}}$ thick, the jump at $80^{\circ} \mathrm{C}$ is hardly observed with a faint trace in slope at this temperature. For much thicker films, neither jump appears in heating experiments. The thickness dependent complex behavior observed in non-equilibrium condition is considered to be some indication of surface and confined effects, because the characteristic film thicknesses coincide with the order of $R_{\mathrm{g}}$.

KEY WORDS: Ultrathin Polystyrene Film / Glass Transition / Thickness Jump / X-Ray Reflectivity /
\end{abstract}

Physical properties of systems are strongly affected by symmetry, dimensionality, and the number of constituent particles. Part of such dependences comprises an interesting field of physics, widely known as confined effects. Numerous experimental and theoretical studies have reported many intriguing confined effects on the glass transition of polymeric materials, and therefore glass-forming polymers are recognized to be an excellent example of such systems. As reproducible experimental results are easily obtained, polystyrene (PS) thin films with atomically flat surfaces have been used as standard glass-forming polymer films. For substrate-supported PS films, a large reduction in the glass transition temperature $\left(T_{\mathrm{g}}\right)$ dependent on the film thickness is observed for films less than $40 \mathrm{~nm}$ thick. ${ }^{1,2}$ More interestingly, the so-called free-standing PS films showed $T_{\mathrm{g}}$ reduction much larger than those of supported films. ${ }^{3,4}$ Since the free-standing PS films are free from the interaction between the solid substrates, the strong reduction in $T_{\mathrm{g}}$ is considered to reflect enhanced mobility inherent in the surface region exposed to air. The positron annihilation technique was used to estimate the free volume in the surface region, and indicated that the effective $T_{\mathrm{g}}$ of PS was $T_{\mathrm{g}}$ (bulk) $-25^{\circ} \mathrm{C}$ and $T_{\mathrm{g}}$ (bulk) $-43{ }^{\circ} \mathrm{C}$ for the surface region to a depth of $5 \mathrm{~nm}$ and $2 \mathrm{~nm}$, respectively., 5 Scanning viscoelasticity microscopy ${ }^{7}$ showed enhanced mobility on the PS surface, and subsequent atomic force microscopy measurements confirmed a soft surface region 3-4 nm thick with low $T_{\mathrm{g}}{ }^{8}$ Regarding thermodynamical considerations, concentration of the ends of molecules in the surface region was pointed out, ${ }^{9}$ suggesting a decrease in polymer density on the surface, subsequently confirmed by simulations. ${ }^{10,11}$ Since the reduced density naturally leads to an increase in the free-volume and enhanced mobility of chain segments, several experimental studies on the surface glass transition of PS successfully employed a two-layer ${ }^{1}$ or three-layer ${ }^{5}$ model where the surface layer is characterized by constant thickness and peculiar physical properties (e.g., density, and viscosity), independent of the bulk properties.

Although arguments based on the increment in the freevolume near the surface fairly describe the glass transition on the surface of PS, another mechanism might be required for films with thicknesses less than or equal to two times the radius of gyration $\left(R_{g}\right)$, where the glass transition process would be more related to the microscopic character of molecular chains. ${ }^{3,4}$ Despite the substantial importance of understanding surface effects in polymers, quantitative information is still favored, presumably reflecting the difficulty in observing each molecule in the surface region. To accumulate precise information on novel anomalies peculiar to such ultrathin glass-forming layers and to elucidate the microscopic nature of molecular chains confined under the space comparable to their dimension, we characterized the temperature dependence of parameters describing supported PS films with X-ray reflectivity (XR), which affords the film thickness within a precision of $0.03 \mathrm{~nm}$. From the interference fringe pattern obtained by $\mathrm{XR}$ with increasing the sample temperature, we deduced tiny jumps in the thickness reproducibly observed at $80^{\circ} \mathrm{C}$ and $92{ }^{\circ} \mathrm{C}$ in films with thicknesses comparable to $R_{g}$. We consider that the thickness dependent jumps in film thickness under nonequilibrium condition come from some kinetics of molecular chains confined in a space with molecular dimension.

\section{EXPERIMENTAL}

Thin PS films were prepared by a spin coating method with Si(100) wafers as substrates. Almost mono-dispersive PS of $M_{\mathrm{w}}=91,000\left(M_{\mathrm{w}} / M_{\mathrm{n}}=1.04, R_{g} \sim 8.4 \mathrm{~nm}\right.$, Polymer Source Inc.) dissolved in toluene was poured onto a rotating $\mathrm{Si}$ wafer $(4,000 \mathrm{rpm})$ capped with a natural oxide layer, which makes the interaction between the substrate and PS relatively weak. ${ }^{1,12,13}$ Prior to XR data acquisition, all samples were annealed at 
$145^{\circ} \mathrm{C}$ for $12 \mathrm{~h}$ before cooling to room temperature in $4 \mathrm{~h}$. PS layers with $\mathrm{Si}$ substrates were cut into several pieces, each with an area of $2 \mathrm{~cm} \times 1 \mathrm{~cm}$.

Specular XR spectra at various temperatures were obtained using a high-resolution four-circle X-ray diffractometer with a rotating-anode X-ray generator (SLX2000+ UltraX, Rigaku Co., Ltd.). Here, $\mathrm{CuK} \alpha_{1}$ radiation (wavelength $\lambda=0.15406 \mathrm{~nm}$ ) was monochromated using a two-bounce $\mathrm{Ge}(220)$ crystal monochromator. As a preliminary measurement, an X-ray illuminated area with a uniform PS layer was searched by $\mathrm{XR}$ at room temperature. Thicknesses evaluated by XR were $4.09 \mathrm{~nm}, 7.43 \mathrm{~nm}, 17.07 \mathrm{~nm}, 26.62 \mathrm{~nm}$, $66.67 \mathrm{~nm}$, and $83.70 \mathrm{~nm}$, corresponding to $0.5 R_{g}, R_{g}, 2 R_{g}$, $3 R_{g}, 8 R_{g}$, and $10 R_{g}$, respectively. $\mathrm{XR}$ was also measured on cooling for another $0.5 R_{g}$ thick film, which is referred to as \#Sample_0.5 $R_{g}$ for cooling. Initial thickness of \#Sample_0.5 $R_{g}$ for cooling at room temperature was $4.34 \mathrm{~nm}$. We will thus call the $0.5 R_{g}$ sample for the heating measurement \#Sample_0.5 $R_{g}$ for heating (the $4.09 \mathrm{~nm}$ thick sample stated above). The fitted root-mean-square surface roughness was found to be less than $0.5 \mathrm{~nm}$ at room temperature. During data collection at high temperatures, samples were kept in dry $\mathrm{N}_{2}$ atmosphere. Temperature was controlled using a homemade heater and a PID temperature controller (Model 134, Cryogenic Control Systems Inc.) with an accuracy of $0.2^{\circ} \mathrm{C}$. The sample temperature was raised by $3{ }^{\circ} \mathrm{C}$ steps at a heating rate $0.3^{\circ} \mathrm{C} /$ min and held at each measured temperature for $30 \mathrm{~min}$ before $\mathrm{X}$-ray irradiation. At each temperature, it took about $120 \mathrm{~min}$ to collect one XR curve. So, roughly speaking, the average heating rate would be $3{ }^{\circ} \mathrm{C} /(3 / 0.3+30+120 \mathrm{~min}) \sim 1.1$ ${ }^{\circ} \mathrm{C} / \mathrm{h}$. XR $I_{\text {Exp }}(i)$ accumulated at a momentum transfer $q_{i}=$ $4 \pi \sin \theta_{i} / \lambda(i=1, M)$ was fitted by a three-layer model, where the calculated XR $I_{\text {cal }}(i)$ was obtained by assuming air, the PS layer, and the Si substrate. Here, $\theta_{i}$ is the $i$-th angle of incidence in each data set, and the typical number of $M$ lies between 300 and 400. Despite the relatively simple layered structure we assumed, the least-square fittings yielded a sufficiently small reliability factor $R\left(=\sum_{1}^{M}\left|I_{\exp }(i)-I_{\text {cal }}(i)\right| / \sum_{1}^{M} I_{\exp }(i)\right)$, which allows us to determine the thickness of the PS layer $h(T)$ with a precision of $0.03 \mathrm{~nm}$ for all the temperatures measured; we found that the value of $R$ was between 0.01 and 0.02 .

\section{RESULTS AND DISCUSSION}

Figure 1 shows a typical example of the temperature dependence of film thickness $h$. The two straight lines are the fitted lines by which $T_{\mathrm{f}}{ }^{\prime}$ (the characteristic temperature under heating, which corresponds to the glass transition temperature usually obtained under cooling, is denoted as ${T_{\mathrm{f}}}^{\prime 14}$ ) of thin films can be defined conventionally as an intersection. The inset shows the thickness dependence of the temperature obtained by this method, which agrees with reported results; ${ }^{1}$ the curves in the inset (fit_1 and fit_2) show the empirical function $T_{\mathrm{f}}{ }^{\prime}(h)=$ $T_{\mathrm{g}}(\infty)\left[1-(A / h)^{\delta}\right]$ widely accepted in the literatures. The curve denoted as fit_1 is calculated with the $T_{g}(\infty)=95.6^{\circ} \mathrm{C}$ (which is also adopted in ref 14), $A=1.85 \pm 0.05 \mathrm{~nm}$, and

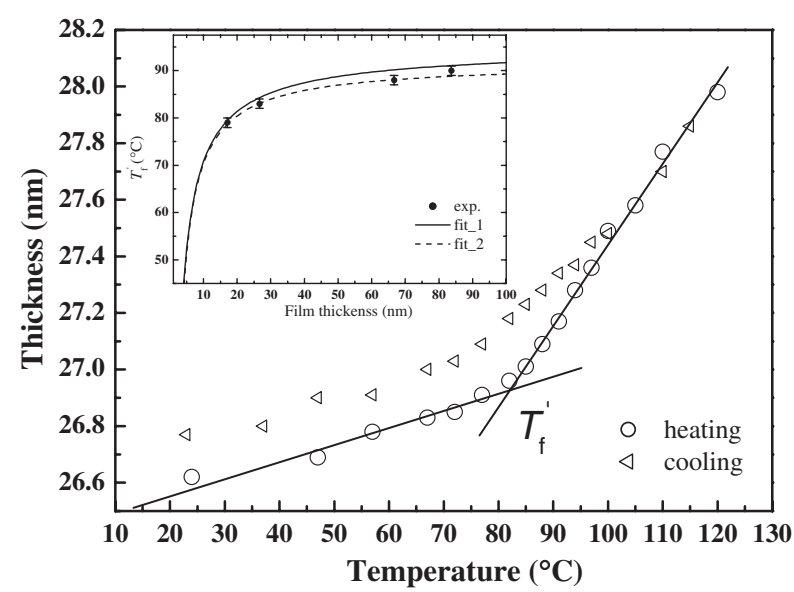

Figure 1. Temperature dependence of the thickness of a PS film with a thickness comparable to $3 R_{\mathrm{g}}$ at room temperature. The open circles and triangles are the points obtained on heating and cooling processes, respectively. Heating and cooling rate was chosen to be $0.3^{\circ} \mathrm{C} / \mathrm{min}$. Inset shows points of intersection of a straight line fitted by using the data below $70^{\circ} \mathrm{C}$ and that above $90^{\circ} \mathrm{C}$ (the temperature is tentatively denoted as $T_{f}^{\prime}$ ). The curves are calculated with the empirical function and fitted parameters (definition and parameters are written in the text).

$\delta=0.8 \pm 0.05$. And the curve denoted as fit_2 is calculated with $T_{g}(\infty)=92{ }^{\circ} \mathrm{C}, A=2 \pm 0.2 \mathrm{~nm}$, and $\delta=0.9 \pm 0.05$.

For thinner films, a small but distinct feature is seen in $h(T)$, as indicated in Figures 2-4. Figure 2(a) shows an XR curve of the film with a thickness corresponding to $0.5 R_{\mathrm{g}}$ (\#Sample_0.5 $R_{g}$ for heating), whereas Figures 3(a) and 4(a) are enlarged portions of the XR curves of films corresponding to $R_{\mathrm{g}}$ and $2 R_{\mathrm{g}}$ thick, respectively. In each $\mathrm{XR}$ profile, the fitted curve is indicated by a solid line, and arrows indicate selected local minima in the fringe pattern due to the interference caused by X-rays reflected at the PS surface and the interface. As clearly displayed by the arrows in Figure 2(a), the positions of local minima of XR show a discontinuous displacement in $q$-value between $77^{\circ} \mathrm{C}$ and $82^{\circ} \mathrm{C}$, above which the minima continuously move to smaller $q$-values indicating a gradual increase in average thickness. The discontinuous jump in $h(T)$ of \#Sample_0.5 $R_{g}$ for heating in the heating is also seen around $80^{\circ} \mathrm{C}$ by closed circles in Figure 2(b). Figures 2(c) and 2(d) depict temperature dependence of surface roughness and electron density of \#Sample_0.5 $R_{g}$ for heating, both of which show reasonable values and tendency with inflections around $80^{\circ} \mathrm{C}$.

In Figure 3(a) and 3(b), another jump in thickness of $0.23 \mathrm{~nm}$ is seen at $92^{\circ} \mathrm{C}$ in the $R_{\mathrm{g}}$ thick film. Furthermore, the jump at $80^{\circ} \mathrm{C}$ seems to disappear in the film of thickness $2 R_{\mathrm{g}}$, although there seems to be faint trace in slope at $80^{\circ} \mathrm{C}$ (Figure 4). For films thicker than $2 R_{\mathrm{g}}$ (in this study, they were $3 R_{\mathrm{g}}, 8 R_{\mathrm{g}}$ and $10 R_{\mathrm{g}}$ thick films), neither jump is observed; the thickness of the $3 R_{\mathrm{g}}$ film happens to be displayed in Figure 1 .

We also tried to observe the jumps under cooling conditions. However, such a jump in film thickness has never been indicated in our measurements. Open triangles in Figure 1 show fitted $h(T)$ corresponding to $3 R_{\mathrm{g}}$ thickness in a sub- 

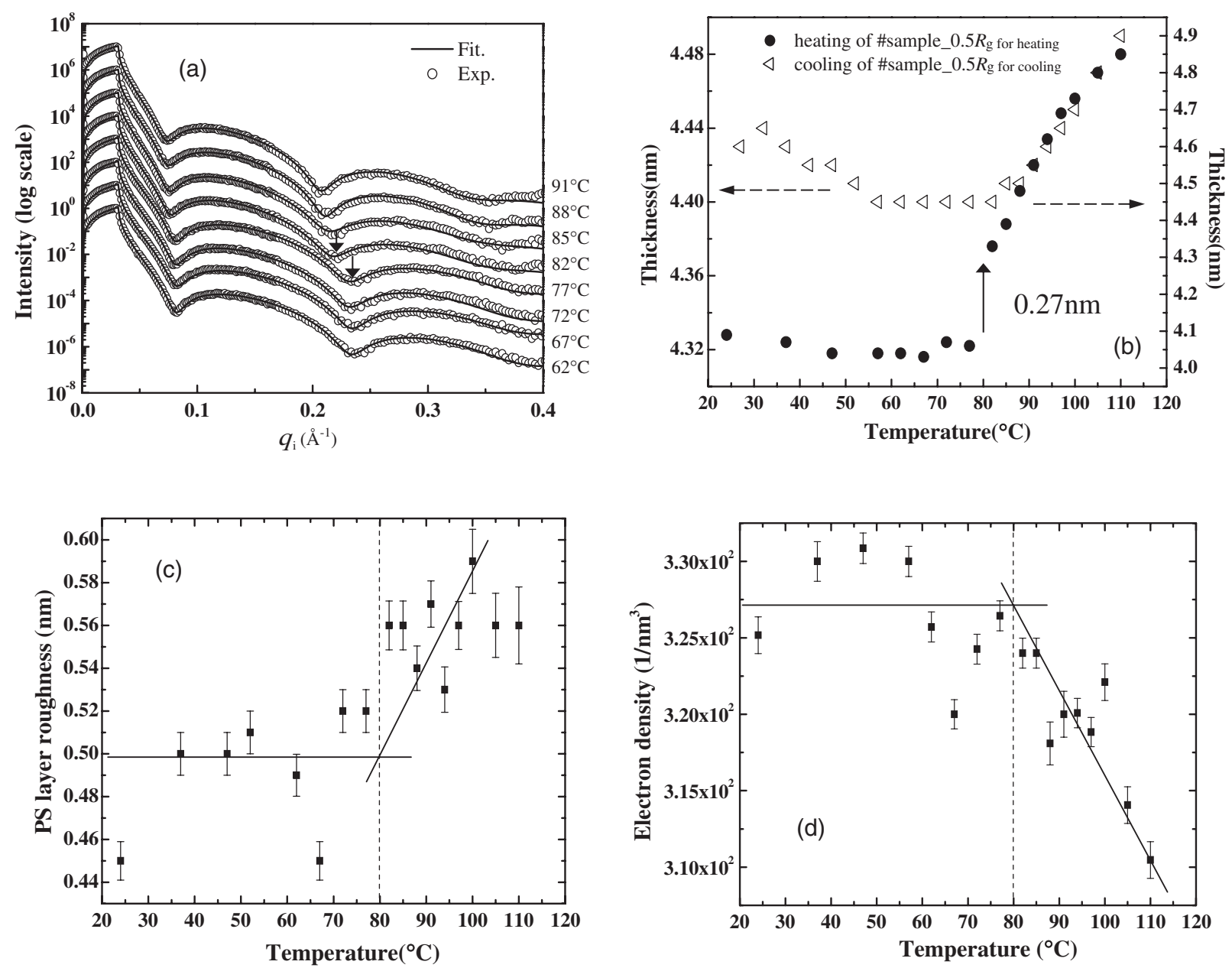

Figure 2. Temperature dependence of X-ray reflectivity profiles of a PS thin film with a thickness comparable to $0.5 R_{\mathrm{g}}(\mathrm{a})$, thickness of the layer $h(T)$ (b), root mean square of surface roughness (c), and electron density (d) obtained by the fittings. For clarity, each XR is shifted vertically. The arrows in (a) show one of local minima in the interference fringe pattern around $80^{\circ} \mathrm{C}$. Since the errors in $h(T)$ is as small as the size of symbols in (b), the error bars are not shown here. Except for the open triangles in (b), results for \#Sample_0.5R $R_{g}$ for heating are shown. In (b), closed circles represent thicknesses of \#Sample_0.5R for heating obtained on heating. For \#Sample_0.5 $R_{g}$ for heating, thickness scale is indicated by the right axis. Whereas open triangles are the thicknesses of \#Sample_0.5Rg for cooling obtained on cooling with thickness of $4.49 \mathrm{~nm}$ at $110^{\circ} \mathrm{C}$. For \#Sample_0.5R $R_{g}$ for cooling, thickness scale is indicated by the left axis.

sequent cooling with the cooling rate of $0.3^{\circ} \mathrm{C} / \mathrm{min}$, of which value is the same as the heating rate. The glass transition temperature $T_{\mathrm{g}}$ obtained in the cooling may slightly be higher than the fictive temperature $T_{\mathrm{f}}{ }^{\prime}$ determined in the heating. $h(T)$ on heating and that on cooling clearly indicate that the supported PS films with thicknesses of the order of $R_{\mathrm{g}}$ never experience thermal equilibrium at lower temperature especially far below $T_{\mathrm{g}}$. Open triangles in Figure 2(b) show $h(T)$ of \#Sample_0.5 $R_{g}$ for cooling with a cooling rate of $0.3^{\circ} \mathrm{C} / \mathrm{min}$. There is no jump around $80^{\circ} \mathrm{C}$, and linear thermal expansivity of \#Sample_ $0.5 R_{g}$ for cooling above $T_{\mathrm{g}}\left(>82^{\circ} \mathrm{C}\right)$ is estimated as $7.9 \times 10^{-4} /{ }^{\circ} \mathrm{C}$ comparable to the value for thicker films $\left(6.0 \times 10^{-4} /{ }^{\circ} \mathrm{C}\right)$ and that of the bulk PS. ${ }^{15}$

The jumps observed only in the heating condition can be regarded as a non-equilibrium phenomenon and presumably be attributed to a microscopic mechanism where an atomic model would dominantly be utilized. Once we take such a viewpoint, some other results would naturally make sense; thermal expansion inferred from the slope of temperature dependence on thickness is practically zero before the jumps (Figures 3(b), 4(b)) and even negative for the thinnest film investigated (Figure 2(b)), which might show a sharp contrast with the case of thicker film (Figure 1) and with that of bulk PS around the room temperature. ${ }^{16}$ It might reflect an absorption of molecular segments of PS extended to the interface between PS and $\mathrm{SiO}_{2}$ on $\mathrm{Si}$. Since the XR data collection requires long time, our measurements can be classified into measurements under slow heating conditions. Since slower rate would generally produce a larger thermal expansivity in the glassy state, almost zero expansion before the jump in our study indicates retardation of thermal expansion in the glassy state. From the literature, ${ }^{17}$ this type of retardation may frequently be observed on the heating process, because the heating process is often started from the state deeply deviated from an equilibrium state. Although origin of two jumps is still unclear to the authors, the jump around $80^{\circ} \mathrm{C}$ may relate with expansion of molecular segments 

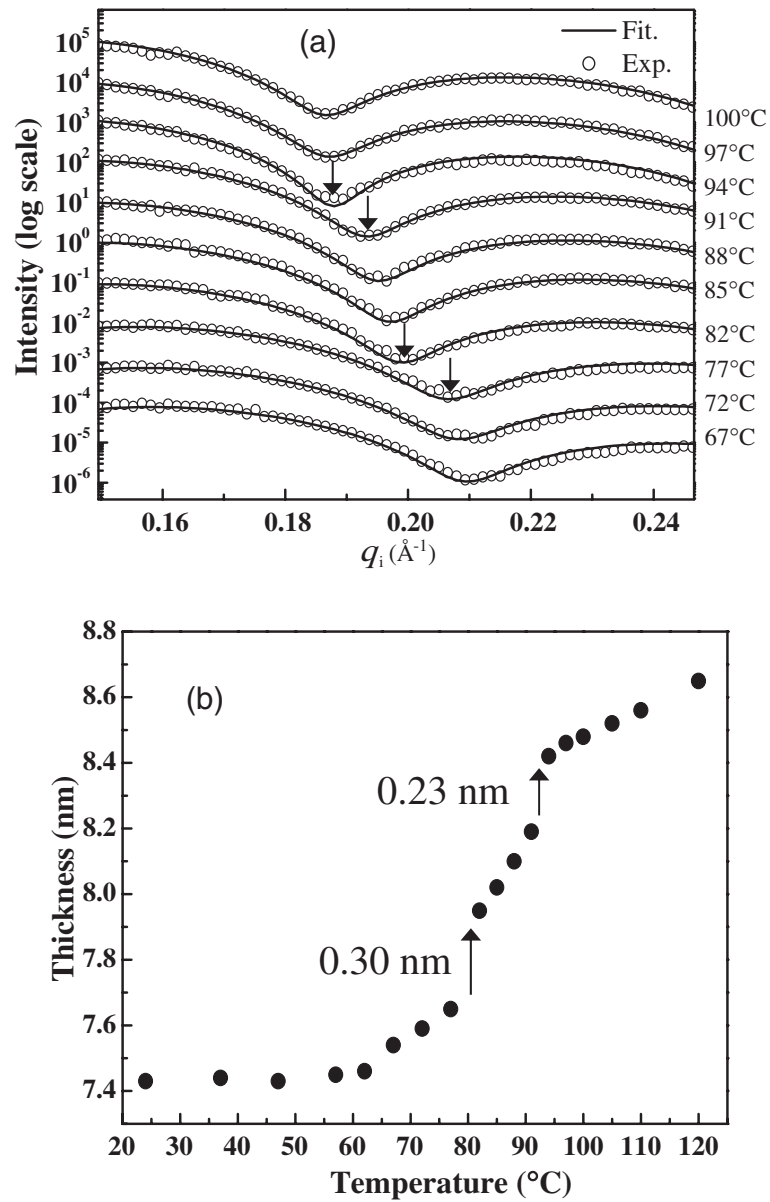

Figure 3. Enlargement of the XR (a) and fitted $h(T)$ (b) of PS with a thickness comparable to $R_{\mathrm{g}}$. For clarity, each $\mathrm{XR}$ is shifted vertically. Two abrupt variations in layer thickness are recognized at $80^{\circ} \mathrm{C}$ and $92^{\circ} \mathrm{C}$.

exposed to the air assisted by higher mobility inherent in the surface region. The jump in the ultrathin films may correspond to the temperature-independent surface layer mainly postulated for thicker films. ${ }^{18}$ If we expand such a speculation, the jump around $92^{\circ} \mathrm{C}$ may arise from the bulk-like layer in the middle of the films. With increasing film thickness, only the jump around $92^{\circ} \mathrm{C}$ would be emphasized compared with the jump around $80^{\circ} \mathrm{C}$ coming from the weakly temperature-dependent surface region. As the thickness further increases, the abrupt jump around $92{ }^{\circ} \mathrm{C}$ would also become less and less noticeable, because fraction of molecular segments in the interfacial region bound to the substrate as well as the fraction in the surface region decreases. If such speculation has some validity to a certain extent, the jump around $80^{\circ} \mathrm{C}$ would depend on several parameters describing the surface morphology, e.g., surface roughness, as well as on intrinsic parameters of PS like $R_{g}$. Furthermore, the $92{ }^{\circ} \mathrm{C}$ jump may be a substrate-dependent phenomenon. Both of them would be open problems for the origin of the jumps to be investigated in near future. Since the mobility of molecular chains at a temperature under heating would not differ from that under cooling so much, we might bring up a microscopic model to explain the anomalies seen
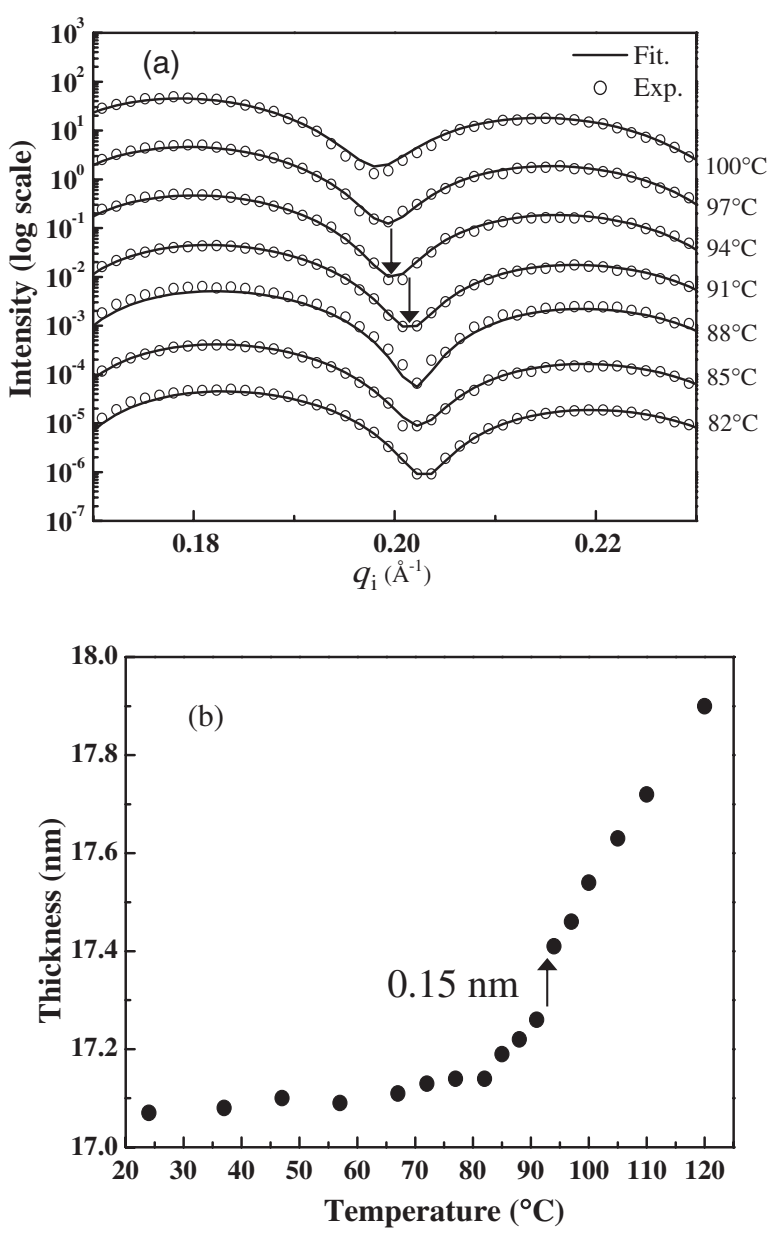

Figure 4. Enlargement of the XR (a) and fitted $h(T)$ (b) of PS layer with a thickness comparable to $2 R_{\mathrm{g}}$. Only one jump of $0.15 \mathrm{~nm}$ is observed around $92^{\circ} \mathrm{C}$.

only in heating. As an example of semi-microscopic model for thin polymer films, the sliding motion proposed in ref 19 would be one of such candidates to which the confined geometry and probability producing irreversibility is appropriately incorporated. Figure 5 shows schematic diagram of a polymer chain confined to a thin film with thickness less than $2 R_{g}$. The molecular chain touching the free surface at the points $\mathrm{A}$ and $\mathrm{B}$ forms a loop-like segment (loop $\mathrm{AB}$ ). Whereas the segment between $\mathrm{A}$ and $\mathrm{B}^{\prime}$ can be regarded as a bridge-like configuration across the film (bridge $\mathrm{AB}^{\prime}$ ). ${ }^{19,20}$ In Figure 5, several dash dotted lines parallel to the two interfaces divide the film into several pseudolayers where the local $T_{\mathrm{g}}$ 's in each pseudolayer increases with increasing distance from the free surface as in a pseudolayer model proposed in ref 21. During the sliding motion considered to be dominant in ultrathin films, source of the enhanced driving force for the sliding motion may be in the loop $\mathrm{AB}$ embedded in the unfrozen pseudolayers at a temperature the inner pseudolayers are still frozen. And bridge $\mathrm{AB}^{\prime}$ may be responsible for the enhanced motion of molecular segments in the pseudolayers near the substrate. Both would become more dominant as the thickness decreases to the order of $R_{\mathrm{g}}$ and the sliding motion of the segment may be mediated 


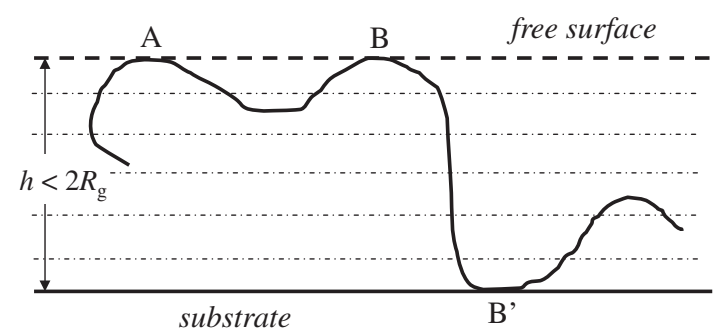

Figure 5. Schematic representation of a polymer chain confined into an ultrathin polymer film with thickness less than $2 R_{g}$. The segment between $A$ and $B$ forms a loop-like configuration, while the segment between $A$ and $B^{\prime}$ can be regarded as a bridge across the free surface and the interface between the polymer and the substrate. Dash-dotted lines represent the boundaries among the pseudolayers where local $T_{\mathrm{g}}$ ' in each pseudolayer is supposed to increase with the distance from the free surface.

toward the free surface at relatively low temperatures below $T_{\mathrm{g}}$, presumably causing the sudden increase in the thickness. Once the major portion of molecular chain is fully exposed and relaxed in the surface region at high temperatures, it might be somewhat difficult for such a chain to find a way to go back the initial configuration under cooling. The simultaneous reduction in polymer density and increase in surface roughness accompanied with the jumps probably serve to specify the type of rearrangements of molecular segments in the ultrathin glassy polymer films.

\section{CONCLUSIONS}

Precise X-ray reflectivity measurements on the temperature dependence of ultrathin polystyrene (PS) films with different thicknesses have been performed. Tiny but reproducible jumps in thickness are found at $80^{\circ} \mathrm{C}$ and $92^{\circ} \mathrm{C}$ for films with a thickness less than three times the radius of gyration. The two jumps suggest that the glass transition temperatures of the surface and bulk-like regions are about $80^{\circ} \mathrm{C}$ and $92^{\circ} \mathrm{C}$, respectively. The thickness dependence of the jumps leads to a better understanding of ultrathin glassy polymer films for confined molecular chains.

Acknowledgment. We are greatly indebted to $\mathrm{Dr} \mathrm{K}$. Shimizu, Professors H. Terauchi and S. Sawada of Kwansei Gakuin University for valuable discussion and critical reading of the manuscript. Part of this study was financially supported by the Promotion and Mutual Aid Cooperation for Private
Schools of Japan (the Science Research Promotion fund in 2006-2008).

Received: March 25, 2009

Accepted: August 7, 2009

Published: September 28, 2009

\section{REFERENCES}

1. J. L. Keddie, R. A. L. Jones, and R. A. Cory, Eur. Phys. Lett., 27, 59 (1994).

2. a) J. A. Forrest and R. A. L. Jones, in "Polymer Surface, Interfaces, and Thin Films," A. Karim and S. Kumar, Ed., World Scientific, Singapore, 2000, pp 251-294.

b) G. Reiter and J. A. Forrest, Eur. Phys. J. E: Soft Matter Biol. Phys., 8, 101 (2002).

3. J. A. Forrest, K. Dalnoki-Veress, J. R. Stevens, and J. R. Dutcher, Phys. Rev. Lett., 77, 2002 (1996).

4. J. A. Forrest, K. Dalnoki-Veress, and J. R. Dutcher, Phys. Rev. E: Stat., Nonlinear, Soft Matter Phys., 58, 6109 (1996).

5. G. B. DeMaggio, W. E. Frieze, and D. W. Gidley, Phys. Rev. Lett., 78, 1524 (1997).

6. Y. C. Jean, R. Zhang, H. Cao, J. Yuan, and C. Huang, Phys. Rev. B: Condents. Matter Mater. Phys., 56, R8459 (1997).

7. N. Satomi, A. Takahara, and T. Kajiyama, Macromelcules, 32, 4474 (1999).

8. J. H. Teichroeb and J. A. Forrest, Phys. Rev. Lett., 91, 016104 (2003).

9. A. M. Mayes, Macromolecules, 27, 3114 (1994).

10. M. Müller, K. Binder, and W. Oed, J. Chem. Soc., Faraday Trans., 91, 2369 (1995).

11. Y. Rouault, B. Dunweg, J. Baschnagel, and K. Binder, Polymer, 37, 297 (1996).

12. J. L. Keddie, R. A. L. Jones, and R. A. Cory, Faraday Discuss., 98, 219 (1994).

13. J. A. Forrest, K. Dalnoki-Veress, and J. R. Dutcher, Phys. Rev. E: Star., Nonlinear, Soft Matter Phys., 56, 5705 (1997).

14. P. Badrinarayanan, W. Zheng, Q. Li, and S. L. Simon, J. Non-Cryst. Solids, 353, 2603 (2007).

15. T. Miyazaki, K. Nishida, and T. Kanaya, Phys. Rev. E: Star., Nonlinear, Soft Matter Phys., 69, 061803 (2004).

16. R. Greiner and F. R. Schwarzl, Rheol. Acta, 23, 378 (1984).

17. J. H. van Zanten, W. E. Wallace, and W. Wu, Phys. Rev. E: Star., Nonlinear, Soft Matter Phys., 53, R2053 (1996).

18. S. Kawana and R. A. L. Jones, Eur. Phys. J. E: Soft Matter Biol. Phys., 10, 223 (2003).

19. P. G. de Gennes, Eur. Phys. J. E: Soft Matter Biol. Phys., 2, 201 (2000).

20. K. Dalnoki-Veress, J. A. Forrest, P. G. de Gennes, and J. R. Dutcher, J. Phys. IV France, 10, Pr7 (2000).

21. M. Bhattacharya and M. K. Sanyal, Phys. Rev. E: Star., Nonlinear, Soft Matter Phys., 71, 041801 (2005). 